\title{
Karl-Hofmann-Publikations-Preis für Dissertationen 2001
}

Die Verleihung des Karl-Hofmann-Publikations-Preises für Dissertationen im Jahr 2001 fand am 2. Oktober 2001 am Institut für Sport und Sportwissenschaft der Universität Karlsruhe statt. Susanne TITLBACH (Uni Karlsruhe), Harald SEELIG (Uni Freiburg) und Stephan WASSONG (DSHS Köln) sind die Preisträger. Die Preise verlieh Thomas Hecht vom Verlag Karl Hofmann, Prof. Dr. Heinz Mechling hielt die Laudationes.

Den Ersten Preis (DM 1500.- sowie die Veröffentlichung der Arbeit in der Schriftenreihe "Beiträge zur Lehre und Forschung im Sport") gewann Susanne TITTLBACH mit dem Dissertationsthema "Entwicklung der körperlichen Leistungsfähigkeit im mittleren und späteren Erwachsenenalter eine prospektive Längsschnittstudie". Den Zweiten Preis (DM 1000.-) erhielt Harald SeELIG mit dem Dissertationsthema "Subjektive Theorien über Laborsituationen. Methodologie und Struktur subjektiver Konstruktionen von Sportstudierenden"; der Dritte Preis (DM 500.-) ging an Stephan WASsONG mit dem Dissertationsthema "Pierre de Coubertins US-amerikanische Studien und ihre Bedeutung für die Analyse seiner frühen Erziehungskampagne".

Der Karl-Hofmann-Publikations-Preis wird vom Verlag Karl Hofmann auf Anregung aus dem Redaktionsausschuss des Verlags, der die beiden Schriftenreihen "Beiträge zur Lehre und Forschung im Sport" und "Texte Quellen - Dokumente zur Sportwissenschaft" betreut, seit dem Jahr 1995 alle zwei Jahre in Höhe von insgesamt 1500.- $€$ vergeben. Mit dem Preis wird das Ziel verfolgt, wissenschaftlich hochwertige Arbeiten junger Wissenschaftler und Wissenschaftlerinnen aus dem gesamten Bereich der Sportwissenschaft zu würdigen. Die Voraussetzungen für die Bewerbung sind eine Dissertation mit Prädikatsnote und die Befürwortung der Gutachter. Das Auswahlverfahren findet jeweils in einem zweistufigen Prozess statt. Im ersten Schritt werden Gutachten zu den einzelnen Arbeiten durch Mitglieder des Redaktionsausschusses und/oder externe Gutachter verfasst; auf der Basis dieser Gutachten werden drei Bewerber/innen ausgewählt. Im zweiten Schritt werden die Dissertationen der drei Bewerber/innen von einem externen, nicht an den Einzelgutachten beteiligten Gutachter vergleichend bewertet. Dieser Gutachter legt daraufhin eine Rangfolge fest. Der Redaktionsausschuss fällt auf der Grundlage des vergleichenden Gutachtens die Entscheidung. 